\title{
Erratum to: A Meta-analysis of the Diagnostic Accuracy of Autism Diagnostic Observation Schedule Module-1 for Autism Spectrum Disorders
}

\author{
Sherab Tsheringla K K. A. Minju • Sushila Russell • Priya Mammen • \\ Paul Swamidhas Sudhakar Russell • M. K. C. Nair
}

Published online: 18 December 2014

(C) Dr. K C Chaudhuri Foundation 2014

\section{Erratum to: Indian J Pediatr DOI 10.1007/s12098-014-1627-9}

The original article contained inadvertent errors. There was reversal of Sensitivity and Specificity values during analyses and thus, has changed the result. Below is the corrected version.

\begin{abstract}
Results A total of 7 cross-sectional studies provided data on 4057 children. The pooled Sn, Sp, DOR and SROC-AUC for the overall diagnostic accuracy of $\mathrm{ADOS}^{\mathrm{M} 1}$ were: $0.91(95 \%$ $\mathrm{CI}=0.89$ to 0.93$), 0.73$ (95\% $\mathrm{CI}=0.69$ to 0.76$), 44.20$ ( $95 \%$ $\mathrm{CI}=15.89$ to 122.95$)$ and 0.90 respectively.

Conclusions It is concluded that $\mathrm{ADOS}^{\mathrm{M} 1}$ with the original diagnostic algorithm has the overall diagnostic accuracy and pooled specificity suggesting moderate accuracy. The pooled sensitivity is high to be used as a screening test for Autism Spectrum Disorders. $\operatorname{ADOS}^{\mathrm{M} 1}$ with the revised diagnostic algorithm should be used for diagnostic purpose.
\end{abstract}

\section{Results}

Pooled sensitivity ( $95 \% \mathrm{CI}, \mathrm{I}^{2}$ for heterogeneity) for detecting DSM-IV/IV-TR or best estimate diagnosis of ASD was 0.91

The online version of the original article can be found at http://dx.doi.org/ 10.1007/s12098-014-1627-9.

S. Tsheringla $\cdot$ K. A. Minju $\cdot$ S. Russell $\cdot$ P. Mammen •

P. S. S. Russell $(\bowtie)$

Child and Adolescent Psychiatry Unit, Department of Psychiatry,

Christian Medical College, Vellore 632 002, Tamil Nadu, India

e-mail: russell@cmcvellore.ac.in

M. K. C. Nair

Child Development Centre, Thiruvananthapuram Medical College,

Thiruvananthapuram, Kerala, India
(95\% CI=0.89 to $0.93, \mathrm{I}^{2}=92.8 \%$ ). Pooled specificity, calculated using data from all the studies, was 0.73 ( $95 \% \mathrm{CI}=0.69$ to $0.76, \mathrm{I}^{2}=90.3 \%$ ). Figure 2 shows the Forest plots for the pooled sensitivity and specificity. The pooled Diagnostic Odds Ratio was 44.26 (95\% CI=15.89 to $122.95, \mathrm{I}^{2}=85.3 \%$ ) (Table 2). A graph of the SROC curve of $\mathrm{ADOS}^{\mathrm{M} 1}$ showing the sensitivity (true-positive rates) vs. specificity (false-positive rates) from individual studies is shown in Fig. 3. The Area Under the Curve (AUC-SROC) was 0.90. Similarly, the data showed that the maximum joint sensitivity and specificity (the Q-value) was also 0.82 and thus was close to the diagonal line of the SROC curve.

As the heterogeneity scores associated with the pooled diagnostic accuracy parameters was significant $\left(\mathrm{I}^{2}\right.$ ranged from 95-92 \%), the dichotomized QUADAS-2 scores representing the quality of the study and sample size was regressed against the DOR (effect size) as the dependent variable as planned. The pooled DOR value of 44.26 (95\% $\mathrm{CI}=15.891$ to $\left.122.95, \mathrm{I}^{2}=85.3 \%\right)$ in the bivariate analysis remained the same after adjusting for the sample size $[\mathrm{RDOR}=1(95 \% \mathrm{CI}=0.99$ to 1$) ; P=0.8]$ and quality of the studies [with the $\mathrm{RDOR}=0.22(95 \% \mathrm{CI}=0$ to 38.47$) ; P=0.4$ ] was adjusted in the meta-regression demonstrating the modifying effect of these two factors on the diagnostic accuracy of $\operatorname{ADOS}^{\mathrm{M} 1}$.

Pooled DOR for detecting ASD among good-quality studies was $73.42(95 \% \mathrm{CI}=11.16$ to 482.83$) ; \mathrm{I}^{2}=90.4 \%$, Tausquared was 0.92 . Among poor-quality studies the pooled DOR was found to be 29 (95\% CI=8.46 to 99.34); $\mathrm{I}^{2}=$ $78.6 \%$, Tau-squared was only 1.27 . Pooled DOR for detecting ASD among studies with large-sampled studies was 68 (95\% CI $=19.58$ to 237.51 ); $\mathrm{I}^{2}=79.1 \%$, Tau-squared was 1.27. Among small-sampled studies the pooled DOR was found to be 21.09 (95\% CI=5.23 to 84.98); $\mathrm{I}^{2}=82.7 \%$, Tau-squared was 1.08 . 
Fig. 2 The Forest plot for sensitivity and specificity of $\operatorname{ADOS}^{\mathrm{M1}}$ (i) Sensitivity of $\mathrm{ADOS}^{\mathrm{M} 1}$

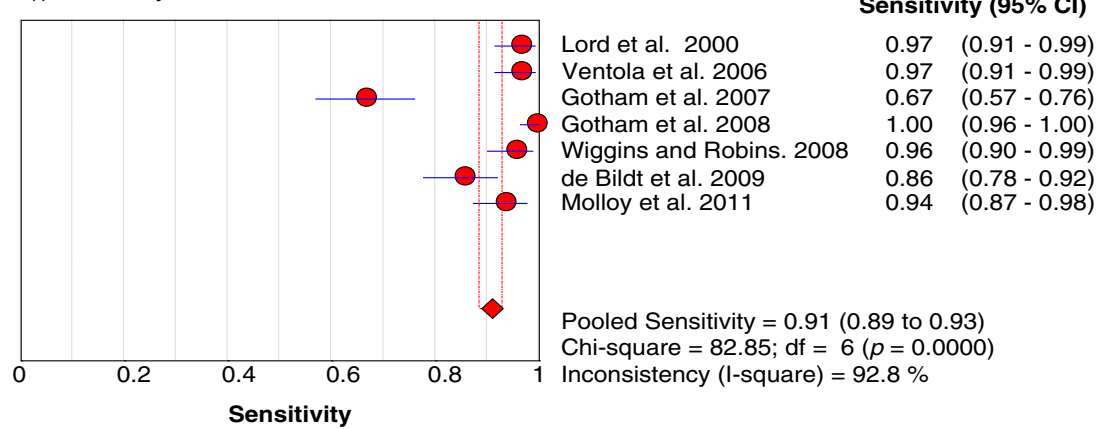

(ii) Specificity of $A D O S^{M 1}$

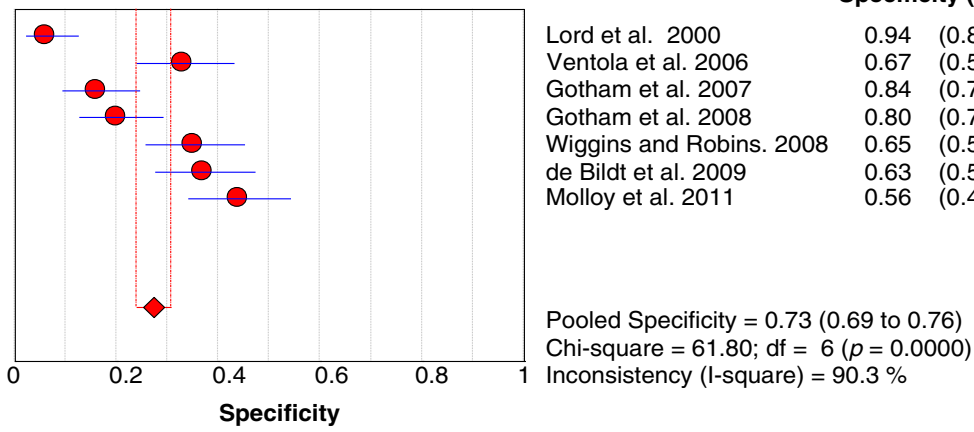

The need to use the $\operatorname{ADOS}^{\mathrm{M} 1}$ with the revised diagnostic algorithm thus becomes a necessity for diagnostic utility. The above stand is supported by the finding that pooled sensitivity and specificity of the $\mathrm{ADOS}^{\mathrm{M} 1}$ were $0.91,0.72$, respectively, indicating a high sensitivity and moderate specificity.

\section{Discussion}

The AUC-SROC for ADOS ${ }^{\mathrm{M} 1}$ in diagnosing ASD was 90. Studies with AUC's values of 0.50 to 0.70 are generally considered of low accuracy, 0.70 to 0.90 as having moderate

Table 2 The summary of the Diagnostic Odds Ratio of $\mathrm{ADOS}^{\mathrm{M} 1}$

\begin{tabular}{lll}
\hline Study & DOR & 95\% CI for DOR \\
\hline Lord et al. 2000 & 506.56 & $123.10-2084.5$ \\
Ventola et al. 2006 & 65.646 & $19.338-222.85$ \\
Gotham et al. 2007 & 10.659 & $5.411-20.996$ \\
Gotham et al. 2008 & 789.29 & $47.014-13251.0$ \\
Wiggins and Robins 2008. & 44.571 & $15.116-131.42$ \\
de Bildt et al. 2009 & 10.459 & $5.217-20.970$ \\
Molloy et al. 2011 & 19.939 & $7.987-49.779$ \\
Pooled DOR (REM) & $\mathbf{4 4 . 2 0 6}$ & $\mathbf{1 5 . 8 9 4 - 1 2 2 . 9 5}$ \\
\hline
\end{tabular}

accuracy, and those with $\mathrm{AUC} \geq 0.90$ as highly accurate [15]. In addition, the index data showed that the maximum joint sensitivity and specificity (the Q-value) was 0.82 and thus was close to the diagonal line of the SROC curve suggesting that $\mathrm{ADOS}^{\mathrm{M} 1}$ is a moderately to highly informative test.

Also, the diagnostic odds ratio (DOR) calculated from sensitivity and specificity was 44.2 which dropped only insignificantly when adjusted for the quality of the study and the sample size. In theory, the DOR ranges in value from zero to infinity, with higher values indicating better discriminatory performance of the test. The DOR of 44 for $\mathrm{ADOS}^{\mathrm{M} 1}$ implies that it has the possibility of correctly diagnosing cases of ASD by 44-folds. Thus it is far better than any random test at diagnosing ASD, even when adjusted for the quality of the study and the sample size, among young children.

The subgroup analysis showed that the pooled DOR was better with high quality studies than poor quality studies. The index analysis to understand this difference showed that the estimate between the study variance (Tau-squared) was lower among studies in the high quality study group at 0.92 when compared with the poor quality study group at 1.27 , suggesting the heterogeneity in the high quality group was far less compromising the DOR in that group. For a similar reason of heterogeneity, the pooled DOR for the large-sampled studies 
Fig. 3 Summary ROC for ADOS $^{\mathrm{M} 1}$

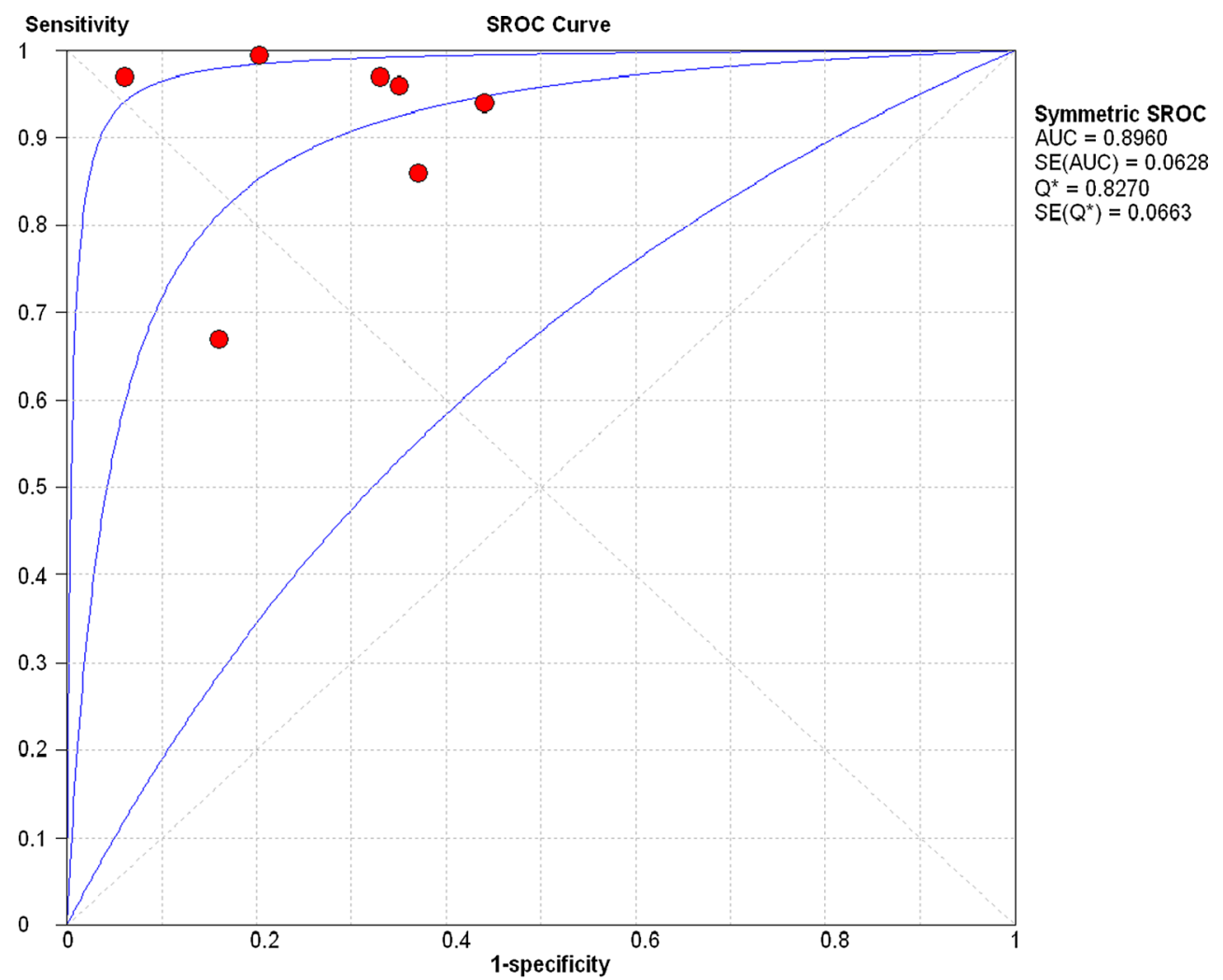

Analysis Options:

Add $1 / 2$ to all cells of the studies with zero

Filter OFF

Symmetric SROC curve fitted using Moses' Model [Weighted regression (Inverse Variance)

Defined relevant region:All ROC spac was better than large-sampled study (Tau-squared for smallsampled study vs. large sampled study $=1.27$ vs. 1.08).

It is concluded, despite the above caveats, that further studies are needed to support the use the original diagnostic algorithm of $\mathrm{ADOS}^{\mathrm{M} 1}$ as the gold standard measure for diagnosing ASD in young children. This meta- analysis raises questions as to whether the $\operatorname{ADOS}^{\mathrm{M} 1}$ cut-off score needs further modification and in the meantime the revised diagnostic algorithm of $\mathrm{ADOS}^{\mathrm{M} 1}$ needs to be used for the confirmation of the diagnosis of Autism Spectrum Disorders in the clinical and educational settings. 